\title{
Assessment of Accelerated Cyclic Electrochemical Technique Compared to Conventional Industrial Testing Methods
}

\author{
Maksim Bahdanchyk $^{1}$, Gianmaria Gasperini ${ }^{2}$, Paolo Gronchi ${ }^{1}$ and Antonello Vicenzo ${ }^{1}$ \\ ${ }^{1}$ Politecnico di Milano, Dipartimento di Chimica, Materiali Ingegneria Chimica "Giulio Natta" , Via \\ Luigi Mancinelli 7, 20131 Milan, Italy \\ ${ }^{2}$ Innovhub - Stazioni Sperimentali per l'Industria S.r.l, Via Giuseppe Colombo 83, 20133 Milan, Italy \\ maksim.bahdanchyk@polimi.it
}

The Accelerated Cyclic Electrochemical Technique (ACET), which has been adopted as the ISO 17463 standard, has attracted great attention in industry as a promising tool for the rapid assessment of paints and organic coatings on metallic substrates. The protocol consists of Electrochemical Impedance Spectroscopy (EIS) and Relaxation Voltammetry (RV) to observe the gradual degradation of a coated sample. The peculiarity of the test lies in the use of cathodic polarization (CP) as a major stress factor allowing a strong acceleration of failure and the quantitative evaluation of both water uptake and coating adhesion, within a limited test time. Indeed, ACET has been reported as a successful method for studying the effect of cure temperature [1], phosphate and ZMP pigments [2,3] and surface pretreatments [4] on the protective properties of painted systems. Moreover, a good agreement with conventional accelerated tests was shown [2,5].

In this work, we confirm that the results of ACET on water uptake correlate well with Humidostatic Chamber (HC) tests performed in accordance to ISO 6270. Conversely, some disagreement is observed with Neutral Salt Spray (NSS) test ISO 9227. As a matter of fact, ACET can reproduce coating performance in NSS only if electrolyte can reach the substrate and cathodic delamination occurs. Accordingly, we highlight that the main limitation of ACET is how quick electrolyte reaches the substrate, so that this technique fails to classify high thickness and/or high resistive coatings.

In the attempt to overcome this intrinsic shortcoming of the test, we propose the modification of ACET by using a sample with a $2 \times 1 \mathrm{~mm}$ crosscut (sACET, where $s$ stands for scribed), i.e. with a similar approach as in the REAP (Rapid Electrochemical Assessment of Paint) test. Results are illustrated in Figure 1 for two different coated systems (S1 and S2) tested in the NSS chamber for $720 \mathrm{~h}$ and by sACET for 6 cycles (inset). The delaminated area around the scratch was measured as 37 and $18 \mathrm{~cm}^{2}$ in NSS, 3.41 and $2.80 \mathrm{~cm}^{2}$ in SACET for S1 and S2, respectively, with a similar trend of increase in both tests. EIS measurements in the sACET protocol allow to track the gradual increase of delaminated area by the variation of chargetransfer resistance $\left(R_{c t}\right)$ before and after subsequent degradation steps as shown in Figure 1c. Therefore, whenever the regular ACET test fails or is not able to provide reliable results, we recommend that scratched samples be used for the assessment of both coating water adsorption and adhesion strength.
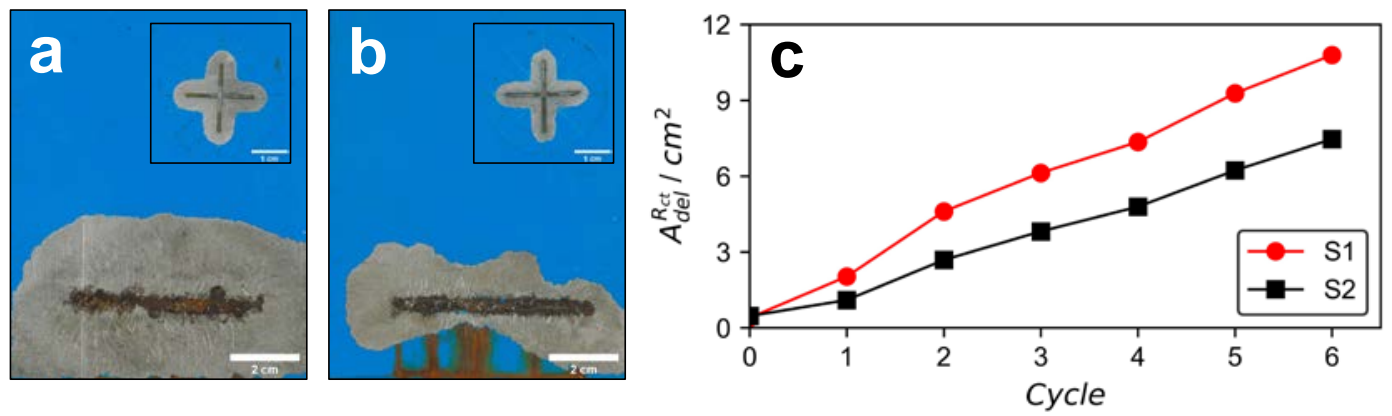

Figure 1 -Coating S1 (a) and S2 (b) after $720 \mathrm{~h}$ in NSS and corresponding SACET samples (in the insets); c) delaminated area evaluated by $\boldsymbol{R}_{c t}$ changes with number of cathodic disbonding cycles.

S.J. García, M.T. Rodríguez, R. Izquierdo, J. Suay, Prog. Org. Coatings. 60 (2007) 303-311. doi:10.1016/J.PORGCOAT.2007.08.002. M.J. Gimeno, S. Chamorro, R. March, E. Oró, P. Pérez, J. Gracenea, J. Suay, Prog. Org. Coatings. 77 (2014) 2024-2030. doi:10.1016/j.porgcoat.2014.04.004. M. Puig, M.J. Gimeno, J.J. Gracenea, J.J. Suay, Prog. Org. Coatings. 77 (2014) 1993-1999. doi:10.1016/J.PORGCOAT.2014.04.031. M.J. Gimeno Pérez, J. Molina Castellanos, R. Izquierdo Escrig, M. Puig Brugal, C. García-García, J.J. Gracenea Zapiraín, J.J. Suay Antón, Mater. Corros. 68 (2017) 1302-1313. doi:10.1002/maco.201609304. M.J. Gimeno, M. Puig, S. Chamorro, J. Molina, R. March, E. Oró, P. Pérez, J.J. Gracenea, J.J. Suay, Prog. Org. Coatings. 95 (2016) 46-53. doi:10.1016/J.PORGCOAT.2016.02.005. 